\title{
Ajuste de un modelo Rasch multidimensional a la escala de respuesta al estrés MNC abreviada
}

\author{
Andrés Burga León \\ Universidad de Lima (Perú)
}

Recibido: 24 de abril del 2009 / Aprobado: 26 de junio del 2009

Se aplicó la Escala de Respuesta al Estrés-MNC a una muestra de 400 estudiantes de una universidad particular de Lima metropolitana, con el fin de construir una versión abreviada de dicha escala. Al ajustar un modelo Rasch de escala de valoración de Andrich, y atendiendo al contenido de cada item, se redujo el tamaño de cada subescala a 12 items. Según los análisis gráficos realizados, estos items median a las personas tan bien como la escala completa y muestran una buena confiabilidad. Todos estos resultados aportan evidencias a favor de la confiabilidad y validez de las inferencias realizadas con las medidas derivadas de la aplicación de la Escala de Respuesta al Estrés MNC-versión abreviada.

análisis Rasch / formas abreviadas / medición de respuesta al estrés

Revision of a multidimensional Rasch model to an abridged Response to Stress Scale MNC

The Stress Response Scale-MNC was applied to 400 students from a private university in Lima Metropolitana, in order to elaborate a short-version of the scale.

The Andrich Rating Scale Rasch model was fitted to the data, and paying attention to the item content, each sub-scale was reduced to 12 items. According to the graphical analysis, this items measure the persons as the full scale does, and have a good reliability. All results gave evidences for the reliability and validity of the inferences made from the measures produced by the Stress Response Scale MNCshort form.

Rasch analysis / short-forms / stress response measurement

Correo electrónico: aburga@ulima.edu.pe 


\section{INTRODUCCIÓN}

El estrés es un fenómeno inevitable en la vida de toda persona y se presenta en mayor o menor medida a lo largo de la vida. Se puede hablar del estrés tanto en el ámbito de la psicología clínica como en el de la psicología educativa y organizacional (Ogden, 1998; Kahn \& Byosiere, 1995; Mandler, 1993). Debe considerarse que son diversas las variables tanto personales como ambientales que intervienen en dicho fenómeno y que la combinación de dichas variables será la que, en última instancia, determine la magnitud de los efectos del estrés sobre una persona.

El término 'estrés' deriva del latín stringere, que significa "provocar tensión". Esta palabra se utilizó por primera vez en el siglo XIV, y a partir de entonces se empleó en diferentes textos en inglés como stress, stresse, strest y streisse. Lazarus \& Folkman (1986) señalan que el término fue empleado a partir del siglo XIV para referirse a experiencias negativas, tales como adversidades, dificultades y tensión, entre otras.

Sin embargo, en el siglo XVII, por influencia del biólogo y físico Robert Hooke, el concepto se asoció a fenómenos físicos como presión, fuerza, distorsión. Sandin (1995) indica que Hooke lo aplicó a estructuras fabricadas por el ser humano que tienen que aguantar el efecto de fuerzas diversas. Stress era la fuerza interna presente en un área sobre la que actúa una fuerza externa (load). Cuando una estructura sólida era distorsionada por fuerzas externas, dicha distorsión era denominada strain y se podía medir en "dinas por centímetro cuadrado" (Sandin, 1995; Lazarus \& Folkman, 1986). Así pues, desde un punto de vista físico, el estrés ha sido definido como una fuerza interna generada dentro de un cuerpo, que se opone a la acción de otra fuerza que tiende a distorsionar dicho cuerpo.

Sandin (1995) señala que a partir de este marco de referencia general, el concepto de estrés va a aplicarse de forma diferente según el sesgo del científico. Así, mientras que los enfoques fisiológicos y bioquímicos consideran el estrés en términos de respuesta, las orientaciones psicológicas y psicosociales, al poner más énfasis en la situación estimular, han tendido a asumir el estrés como un fenómeno externo focalizado en el estímulo. Existen, por lo tanto, dos tipos de perspectivas: las que enfatizan el componente de respuesta fisiológica y las que enfatizan el componente externo (estresor). Posteriormente, se propuso un nuevo componente, que serían los factores psicológicos o subjetivos (cognitivos) que median entre los agentes estresantes y las respuestas fisiológicas de estrés, con lo que se llega a los modelos interaccionales.

Gutiérrez (1998) señala que el término stress, castellanizado como 'estrés', se ha extendido: 
[...] sobrepasada su aplicación biológica inicial, se ha diversificado su análisis y han surgido varias escuelas o corrientes de opinión pertenecientes a disciplinas diversas respecto a cómo encarar la comprensión global de su génesis y desarrollo, su prevención y las posibles intervenciones para la disminución o supresión de sus negativos correlatos fisiológicos, en los casos más graves.

Puede considerarse que el estrés es un fenómeno concomitante a la existencia humana y que, en caso de alcanzar niveles elevados, afecta a las personas y organizaciones de una manera negativa. Por esto resulta importante tener un diagnóstico adecuado del nivel de estrés, de tal manera que se puedan aplicar las estrategias preventivas o correctivas pertinentes. Hablar de "la respuesta de estrés" equivale a decir que la persona se encuentra estresada. Parece que la respuesta de estrés está esencialmente unida a la presencia de algún tipo de respuesta del organismo que es etiquetada como "de estrés" (Sandin, 1995; Valdés $\&$ De Flores, 1990).

Esta respuesta puede dividirse en dos aspectos fundamentales: la respuesta fisiológica y la respuesta psicológica (Ogden, 1998). Ambas facetas de la respuesta (la fisiológica y la psicológica) se encuentran interconectadas y se les puede considerar interdependientes (Sandin, 1995; Lazarus, 1993). La respuesta al estrés puede ser vista dentro del marco de los modelos de la especificidad individuo-respuesta (Lazarus \& Folkman, 1986). El con- cepto de especificidad individuo - respuesta (I-R) supone que una misma persona tiende a responder fisiológicamente de forma similar a diferentes estímulos estresantes. Una única persona emitiría una jerarquía estable de respuestas a diferentes estímulos (Sandin; Chorot; Santed Jiménez, 1995). Esta teoría ha sido postulada por diferentes autores con términos más o menos equivalentes, tales como el de "estereotipia de respuesta vegetativa", presentado por Lacey y Lacey en 1958; "especificidad de respuesta individual", planteado por Engel en 1960; o "especificidad de órgano", presentado por Alexander en 1950 (Lazarus \& Folkman, 1986). El aspecto común de todas estas formulaciones es la noción de que la persona tiende a responder al estímulo estresante con hiperreactividad de alguna modalidad fisiológica.

Para la presente investigación se asumió el modelo de Huber (1980), quien define el estrés como un peligro que amenaza el bienestar e incluso la supervivencia. Cuando la persona se ve afectada, intenta ajustarse a dichas condiciones actuando contra ellas o intentando huir. La amenaza tiene que percibirse como tal, como estímulos aversivos que actúan sobre la persona, que trata de evitarlos, pero muchas veces esto no es posible y debe confrontarlos. Además, considera que todas las reacciones de las personas ante el estrés se pueden clasificar en tres niveles distintos de reacción o niveles de comportamiento: 
- Nivel motor.- Abarca todos los músculos estriados del cuerpo, es decir todos los que están bajo control voluntario. Reaccionan con una tensión muscular que incide en diversas partes del cuerpo.

- Nivel neurovegetativo.- Comprende todas las reacciones del sistema nervioso vegetativo y de los órganos vinculados con este, como los intestinos, las glándulas, etcétera. Los órganos reaccionan con un funcionamiento más intenso, aumenta la circulación, el ritmo cardiaco, la sudoración de la piel, los movimientos peristálticos y los niveles de catecolaminas, entre otras reacciones.

- Nivel subjetivo-cognitivo.- Incluye todos los procesos de pensamiento y de percepción, es decir, la vivencia subjetiva de una situación tal y cómo se la puede expresar verbalmente. Incluye todo lo que podemos decir acerca de nosotros, como sentimientos o situaciones, o cómo comprendemos el mundo desde el punto de vista de las representaciones simbólicas. En este nivel se reacciona con sensaciones emocionales que provocan malestar (como la vergüenza, la inseguridad, el miedo, el pánico) con perturbaciones de la capacidad de atención y de memoria. Hay también respuestas de ansiedad, depresión, insatisfacción con el trabajo y la vida, fatiga, etcétera.
Diversas investigaciones han demostrado por separado la validez de estos indicadores (Skosnik, Chatterton, Swisher \& Park, 2000; Pruessner, Hellhammer \& Kirschbaum, 1999; Keltikangas-Jaervinen, Kettunen, Ravaja, Naeaetaenen, 1999; Verlander, Benedict \& Hanson, 1999; Czeisler \& Richardson, 1998; Daroff \& Martín, 1998; Straus, 1998; Meerlo, Van Den Hoofdakker, Koolhaas \& Daan, 1997; Tuomisto, 1997; Calvo, Szabo \& Capafons, 1996; Spielberger \& Moscoso, 1996; Ficek \& Wittrock, 1995; Sandin, 1995; Selye, 1993; Revelle \& Loftus, 1992).

Siguiendo el modelo de Huber (1980) se construyó la Escala de Respuesta al Estrés MNC (Burga, 2001; Burga, 2003), cuyas propiedades psicométricas son satisfactorias. Posteriormente al análisis de los resultados en esta escala, se puede determinar la estrategia de intervención más adecuada, de acuerdo con el nivel en el cual manifiesta prioritariamente el estrés una persona. Autores como Amutio (1998) señalan que hay estrategias de intervención específicas para los síntomas más bien somáticos de estrés, ya sea que estos se ubiquen sobre todo a nivel del sistema esqueleto motor o a nivel de las reacciones autonómicas; además, habría técnicas de intervención específicas para las reacciones psicológicas. La misma opinión es sustentada por autores como Huber (1980) y Lehrer \& Woolfolk (1993). 
A lo largo de los años se ha utilizado este instrumento en la práctica profesional y en la docencia. Esta experiencia ha permitido constatar que en muchos casos los estudiantes reportan que les resulta algo complicado aplicar el instrumento porque es un poco extenso. Asimismo, en nuestra práctica profesional hemos encontrado que algunas personas también encuentran el instrumento un poco extenso.

Por este motivo, la presente investigación tuvo como objetivo construir una versión abreviada de dicho instrumento, denominada Escala de Respuesta al Estrés MNC - Abreviada (ERE-MNC-A). Las propiedades psicométricas de esta nueva versión fueron analizadas utilizando modelos Rasch.

\section{MetodologíA}

\section{Participantes}

Se trabajó con una muestra no probabilística de tipo intencional, conformada por 400 estudiantes de una universidad privada de Lima metropolitana. Como se utiliza un modelo Rasch, que produce parámetros independientes de las características de la población, no es un problema utilizar muestras no probabilísticas (Muñiz, 1997). Los estudiantes estaban distribuidos según los semestres académicos: 34,8\% correspondían a ciclos iniciales, $32,0 \%$ a ciclos intermedios y $33,3 \%$ a ciclos avanzados. Las edades fluctuaban entre 17 y 30 años ( media $=20,3$; d.e. $=2,4)$, siendo el $50,5 \%$ de sexo femenino y el $49,9 \%$ de sexo masculino.

\section{Instrumento}

La prueba original, denominada Escala de Respuesta al Estrés - MNC (EREMNC) fue construida por Burga (2001) y consta de 65 ítems agrupados de acuerdo con las tres áreas señaladas por Huber (1980). Cada ítem está constituido por una oración que describe algún tipo de reacción ante alguna situación evaluada como estresante por la persona que responde. El formato de respuesta es politómico de cinco puntos: la persona evaluada debe marcar con un aspa la opción que indique la frecuencia con que le ocurre lo señalado en cada una de las oraciones, cuando se enfrenta a una situación considerada estresante.

Como se observa en la tabla 1 , los índices de discriminación en general son aceptables y la confiabilidad de las puntuaciones es buena. No todos los ítems aprobaron el criterio de jueces, pero como se señala en Burga (2001), fueron retenidos porque mostraban un buen funcionamiento empírico. Para obtener datos respecto a la validez de constructo se factorizaron las puntuaciones de las tres subescalas. Se extrajo un solo factor, denominado "respuesta al estrés", que explica el 79,24\% de la varianza. 
Tabla 1

Propiedades psicométricas de la ERE-MNC

\begin{tabular}{lcccc}
\hline Subescala & Número de ítems & ritc & Alpha de Cronbach & $\begin{array}{c}\text { Ítems con V } \\
\text { de Aiken } \\
\text { significativa }\end{array}$ \\
\hline Motora & 22 & $0,38-0,65$ & 0,91 & 20 \\
Neurovegetativa & 21 & $0,14-0,64$ & 0,86 & 17 \\
Cognitivo-afectiva & 22 & $0,31-0,71$ & 0,92 & 20 \\
\hline$n=400$ & & & &
\end{tabular}

$\mathrm{n}=400$

\section{Procedimiento}

Se coordinó con los secretarios académicos de las distintas facultades a fin de poder aplicar el instrumento a los estudiantes. Se garantizó la confidencialidad de los datos, asegurando que no se señalaría el nombre de la universidad o de las facultades que participaron en este estudio. Se ingresó a los salones y se les explicó a los estudiantes que el objetivo de la presente investigación era observar cómo estaba funcionando este instrumento y que la aplicación era anónima. Se les dijo a los estudiantes que su participación era voluntaria y que si alguno no deseaba responder el instrumento, podía retirarse del salón. Solo se retiraron tres estudiantes de uno de los salones.

Luego de recolectada la información, se armó una base de datos en MSExcel. La información fue analizada utilizando los programas Winsteps 3,63 (Linacre, 2006) y ConQuest (Adams, Wu \& Wilson, 1998), debido a que permiten ajustar diferentes modelos Rasch al conjunto de datos analizados.

\section{Resultados}

En una primera etapa se procesó cada una de las tres subescalas de la ERE-MNC por separado, con la finalidad de reducir el número de ítems que la conforman a 12. Esta reducción se llevó a cabo considerando los siguientes criterios:

- Se eliminaron aquellos ítems con contenido redundante, es decir, que se referían al mismo indicador de forma diferente.

- Se eliminaron los ítems que no mostraron un buen ajuste al modelo unidimensional de Escala de Valoración de Andrich (1996). Para ello, se consideró que los índices de infit y outfit debían estar dentro del rango 0,70 - 1,30 (Linacre, 2005).

- El índice de confiabilidad de separación de las personas (equivalente al alpha de Cronbach en el contexto de los modelos Rasch) no debía ser inferior a 0,80 . Este valor se obtuvo considerando los errores estándar del modelo y a todas las personas evaluadas. 
- El conjunto de ítems debían, en esencia, comportarse de modo unidimensional.

- Las medidas establecidas según la versión abreviada no debían diferir significativamente de las medidas establecidas mediante la versión completa.

A continuación, se presentan los ítems seleccionados para cada subescala, que cumplieron con los criterios antes mencionados.

Subescala M (motora):

- Siento dolores en los hombros.

- Siento dolores en la espalda.

- Siento dolores en la nuca.

- Siento los músculos contraídos.

- Siento mi cuerpo tenso y rígido.

- Me tiemblan las manos.

- Siento que mis piernas tiemblan.

- Siento debilidad general.

- Me canso con facilidad.

- Siento que me falta energía para realizar mis actividades.

- Siento que las piernas me pesan.

- Siento calambres en las piernas.

Subescala N (neurovegetativa):

- Sufro de problemas digestivos.

- Me arde la boca del estómago.

- Me despierto varias veces en la noche.

- Me es difícil conciliar el sueño.

- Mis manos sudan.
- Sudo en abundancia.

- Mi corazón late de manera irregular.

- Tengo palpitaciones aceleradas.

- Tengo sensaciones de hormigueo en la piel.

- Siento que mi temperatura corporal cambia.

- Me siento mareado.

- Me falta el aire.

Subescala C (cognitiva-afectiva):

- Me olvido de lo que tengo que hacer.

- Olvido dónde he dejado las cosas.

- No me puedo concentrar en lo que estoy haciendo.

- Me distraigo fácilmente.

- Pienso en cosas que no puedo sacar de mi mente.

- Me es difícil concentrarme en mis actividades.

- Siento que ninguna actividad me interesa en realidad.

- Siento que soy incapaz de enfrentar nuevas responsabilidades.

- Pienso que todo lo que hago está mal.

- Pienso que soy una persona mediocre.

- Me siento frustrado.

- Me siento intranquilo.

Observemos en la tabla 2 los indicadores de confiabilidad y validez: 
Tabla 2

Indicadores de confiabilidad y validez para cada subescala

\begin{tabular}{lccc}
\hline Indicador & Subescala M & Subescala N & Subescala C \\
\hline Confiabilidad de la separación de personas & 0,86 & 0,80 & 0,88 \\
Varianza explicada por la dimensión principal & $60,2 \%$ & $51,5 \%$ & $61,5 \%$ \\
Autovalor del primer componente & 2,2 & 1,9 & 2,2 \\
Varianza del primer componente & $6,2 \%$ & $7,7 \%$ & $7,0 \%$ \\
\hline
\end{tabular}

En cuanto a la confiabilidad, los índices de las tres subescalas fluctúan entre 0,80 y 0,88 . Respecto a la validez, podemos observar que la varianza explicada por la dimensión principal fluctúa entre $51,5 \%$ y $61,5 \%$. Además, los autovalores son pequeños (varían entre 1,9 y 2,2 ).

Como se ve en la tabla 2, los ítems de la versión abreviada se ajustan bien al modelo de Andrich (1993). Una pregunta importante que se hace es si las medidas obtenidas con esta versión abrevia- da no difieren en términos significativos de aquellas estimadas con la escala completa. Para ello, graficamos las medidas obtenidas con ambas versiones en un diagrama de dispersión, trazando los intervalos de confianza según los errores estándar de medición observados para las personas evaluadas. Estos resultados son presentados en las figuras 1 a 3, las cuales indican que, en general, ambas mediciones se encuentran dentro de los márgenes de error.

Figura 1

Comparación de las medidas obtenidas con la versión abreviada y la completa, subescala M

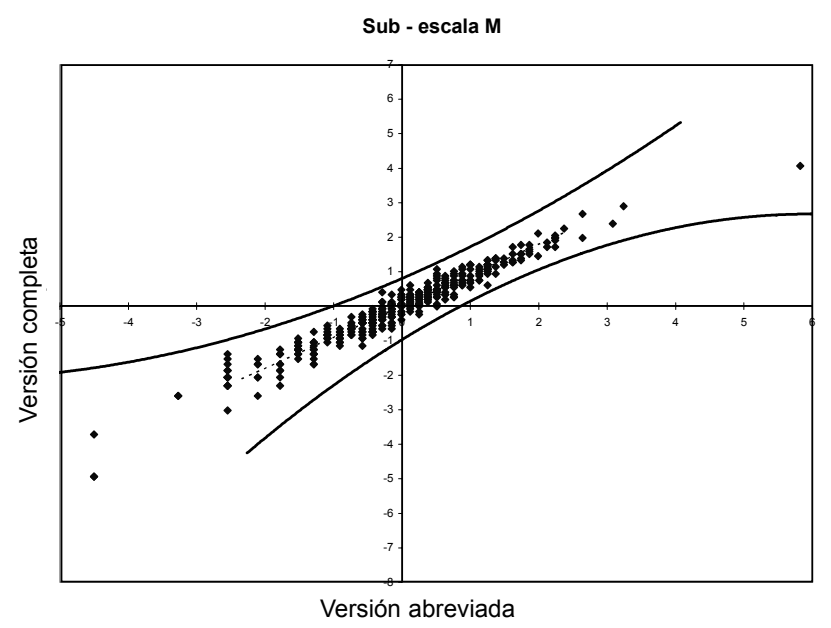


Figura 2

Comparación de las medidas obtenidas con la versión abreviada y la completa, subescala $\mathrm{N}$



Figura 3

Comparación de las medidas obtenidas con la versión abreviada y la completa, subescala $\mathrm{C}$

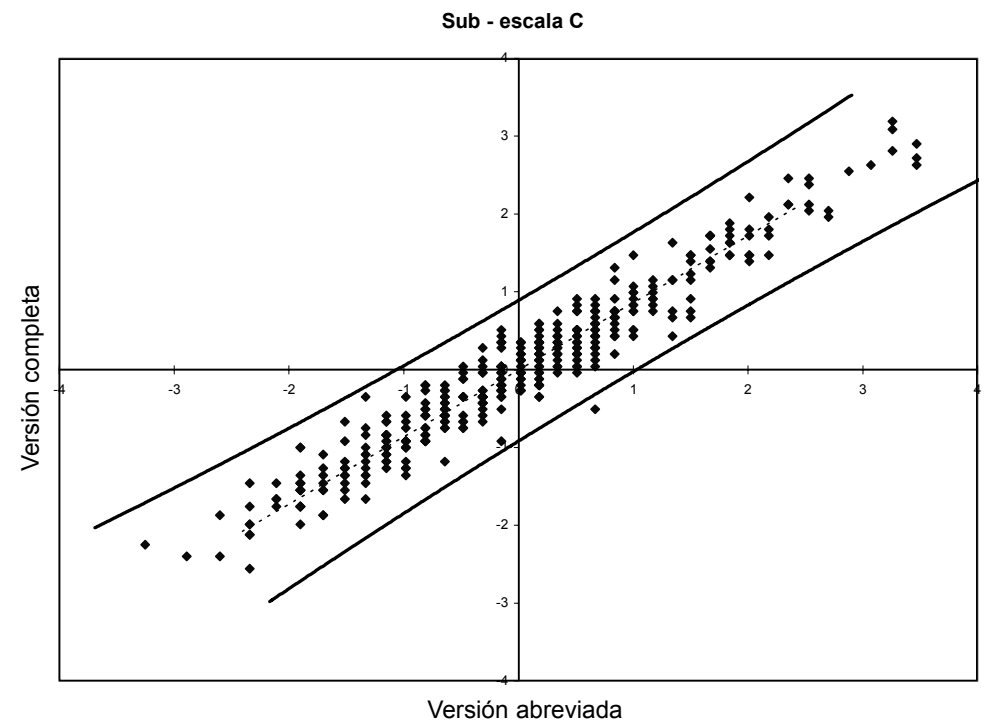


En una segunda etapa se aplicaron los 36 ítems y se ajustó un modelo Rasch multidimensional considerando tres dimensiones. Los indicadores de ajuste para cada ítem se encuentran en un rango aceptable y su dificultad ha sido fijada para que su media aritmética sea 0 . Los resultados son presentados a continuación para cada subescala:

Tabla 3

Medida, error y ajuste de los ítems de la subescala $M$

\begin{tabular}{lrrrr}
\hline ítem & medida & error & infit & outfit \\
\hline M01 & $-0,315$ & 0,037 & 0,63 & 0,67 \\
M02 & $-0,411$ & 0,037 & 1,06 & 1,07 \\
M03 & 0,607 & 0,040 & 1,10 & 1,11 \\
M04 & 0,132 & 0,038 & 0,91 & 0,87 \\
M05 & $-0,377$ & 0,037 & 1,01 & 1,01 \\
M06 & $-0,574$ & 0,037 & 0,97 & 0,96 \\
M07 & 0,699 & 0,040 & 0,83 & 0,82 \\
M08 & $-0,656$ & 0,037 & 0,95 & 0,94 \\
M09 & $-0,545$ & 0,037 & 0,87 & 0,86 \\
M10 & 0,986 & 0,041 & 1,13 & 1,09 \\
M11 & 0,501 & 0,039 & 0,91 & 0,91 \\
M12 & $-0,047^{*}$ & -- & -- & - \\
\hline n $=400$ & \multicolumn{5}{c}{} \\
* El parámetro ha sido fijado, por ello no se calculan \\
el error ni las medidas de ajuste.
\end{tabular}

Tabla 4

Medida, error y ajuste de los ítems de la subescala $\mathbf{N}$

\begin{tabular}{lrrrr}
\hline ítem & medida & error & infit & outfit \\
\hline N01 & $-0,355$ & 0,040 & 0,99 & 0,99 \\
N02 & 0,188 & 0,042 & 1,07 & 1,05 \\
N03 & 0,079 & 0,041 & 1,00 & 0,97 \\
N04 & $-0,392$ & 0,040 & 1,23 & 1,22 \\
N05 & 0,017 & 0,041 & 1,17 & 1,14 \\
N06 & $-0,470$ & 0,040 & 1,32 & 1,29 \\
N07 & $-0,064$ & 0,041 & 1,24 & 1,23 \\
N08 & $-0,481$ & 0,040 & 1,34 & 1,29 \\
N09 & 0,347 & 0,043 & 0,77 & 0,72 \\
N10 & 0,142 & 0,042 & 0,82 & 0,79 \\
N11 & 0,204 & 0,042 & 1,30 & 1,21 \\
N12 & $0,784^{*}$ & -- & -- & - \\
\hline n = 400 & \multicolumn{5}{c}{ Tabla 5 } \\
* El parámetro ha sido fijado, por ello no se calculan \\
el error ni las medidas de ajuste. \\
\multicolumn{5}{c}{. }
\end{tabular}

Medida, error y ajuste de los ítems de la subescala $\mathrm{C}$

\begin{tabular}{lrrrr}
\hline ítem & medida & error & infit & outfit \\
\hline & & & & \\
C01 & 0,188 & 0,037 & 0,82 & 0,82 \\
C02 & $-0,536$ & 0,036 & 0,80 & 0,81 \\
C03 & $-0,656$ & 0,036 & 0,92 & 0,93 \\
C04 & 0,948 & 0,039 & 0,88 & 0,85 \\
C05 & 0,088 & 0,037 & 1,05 & 1,06 \\
C06 & $-0,634$ & 0,036 & 1,01 & 1,02 \\
C07 & $-0,120$ & 0,036 & 1,09 & 1,12 \\
C08 & $-0,748$ & 0,036 & 1,02 & 1,03 \\
C09 & 0,548 & 0,037 & 0,78 & 0,77 \\
C10 & 0,789 & 0,038 & 0,75 & 0,72 \\
C11 & $-0,346$ & 0,036 & 0,72 & 0,73 \\
C12 & $0,481^{*}$ & -- & - & - \\
\hline $\mathrm{n}=400$ & & & &
\end{tabular}

* El parámetro ha sido fijado, por ello no se calculan el error ni las medidas de ajuste. 
Como se ajustó un modelo de Andrich, se presentan a continuación los valores de los umbrales RaschAndrich para nuestra escala multidimensional:

Tabla 6

Umbrales Rasch-Andrich para el modelo multidimensional

\begin{tabular}{lcc}
\hline Umbral & Parámetro & Error \\
\hline 1 & $-1,299$ & 0,018 \\
2 & $-0,763$ & 0,017 \\
3 & 0,807 & 0,026 \\
4 & $1,255^{*}$ & - \\
\hline $\mathrm{n}=400$ & \\
*Los parámetros son restringidos para que su suma \\
sea 0.
\end{tabular}

Finalmente, se obtuvieron diversos estadísticos descriptivos de las tres subescalas estudiadas:

Tabla 7

Correlación, covarianza, media y varianza de las tres subescalas

\begin{tabular}{cccc}
\hline & $\mathbf{M}$ & $\mathbf{N}$ & $\mathbf{C}$ \\
\hline $\mathrm{M}$ & - & 0,515 & 0,439 \\
$\mathrm{~N}$ & $0,85^{*}$ & - & 0,311 \\
$\mathrm{C}$ & $0,74^{*}$ & $0,61^{*}$ & - \\
Media & $-0,969$ & $-1,090$ & $-0,462$ \\
Varianza & 0,707 & 0,516 & 0,499 \\
\hline $\mathrm{n}=400$ & & & \\
${ }^{*} \mathrm{p}<0,001$ & & &
\end{tabular}

Sobre la diagonal se encuentran las covarianzas entre las escalas; debajo de la diagonal presentamos las correlaciones entre ellas. Estas escalas fueron posteriormente factorizadas utilizando el método de eje principal. Se obtuvo un único factor con un autovalor superior a 1,00. Dicho factor explica el $60,7 \%$ de la varianza.

\section{DISCUSIÓN}

Cada una de las subescalas por separado muestra un buen ajuste al modelo de Andrich, lo cual implica que las mediciones derivadas de ellas pueden ser consideradas confiables y válidas. Es decir, el error de medición cometido no es muy grande, pues presentan valores superiores a 0,80 (Bond, 2003).

$\mathrm{Al}$ analizar la unidimensionalidad de las subescalas se empleó el análisis de componentes principales de los residuos (Linacre, 2005). Las tres mostraron una buena proporción de varianza explicada por la dimensión principal (por lo menos el 50\%). El autovalor del primer componente resulta ser 2,2 en el peor de los casos, es decir, la presencia de una posible dimensión secundaria tiene como máximo la fuerza de dos ítems. Consideramos que este tamaño es demasiado pequeño como para construir otra escala independiente con dos ítems. Estos resultados implican que se está midiendo un constructo unidimensional con cada una de las subescalas. Este constructo unidimensional cuenta, además, con validez de contenido según el criterio de jueces (Burga, 2001).

En general, el modelo estimado de tres dimensiones ha mostrado un buen ajuste de los ítems. Todos ellos se encuentran dentro del rango aceptable de 
0,50 a 1,50 (Linacre, 2005), estando la mayoría de ellos entre 0,70 y 1,30 . Esto quiere decir, según Bond (2003), que los ítems se están comportando según las predicciones del modelo psicométrico, lo cual aporta evidencia a favor de la validez de los datos. Como señala ese mismo autor, la invarianza de los parámetros estimados para los ítems y las personas, que se logra cuando un conjunto de datos se ajusta adecuadamente a un modelo Rasch, es la esencia de la validez en las ciencias humanas. Esto quiere decir que logramos medidas de la respuesta al estrés que no dependen ni de las características de las personas evaluadas, ni del conjunto de ítems que responde la persona.

Los umbrales Rasch-Andrich muestran valores ordenados de menor a mayor. Esto quiere decir que todas las categorías resultan modales según los diversos niveles de la variable latente (Linacre, 2001; Shaw, Wright \& Linacre, 1992). Esta es también una evidencia de la validez de la escala de valoración utilizada, pues las personas que responden la prueba pueden discriminar adecuadamente las distintas opciones de esta escala politómica de respuesta (Andrich, 1996).

Como se mostró con el análisis factorial a nivel de subescalas, las tres parecen estar midiendo un único factor, es decir, se comportan unidimensionalmente. Esta dimensión, que se encuentra en la base de las tres subescalas, sería el constructo "respuesta al estrés".
Si bien las muestras no probabilísticas pueden traer muchas complicaciones al momento de realizar inferencias a la población, esto no resulta un problema al momento de estimar los parámetros de los ítems de una prueba con modelos Rasch (Muñiz, 1997). Sin embargo, no se han propuesto baremos en función de esta muestra no probabilística y se invita a otros investigadores a realizarlos usando muestras probabilísticas.

En conclusión, los análisis realizados demuestran que esta escala abreviada se ajusta bien a un modelo Rasch multidimensional y que sus mediciones no se alejan significativamente de aquellas derivadas de la versión completa.

\section{REFERENCIAS}

Amutio, A. (1998). Nuevas perspectivas sobre la relajación. Bilbao: Desclee de Bouwer.

Andrich, D. (1996). Category ordering and their utility. Rasch Measurement Transaction, 9 (4), 464. [En línea] http://rasch.org/rmt/rmt94f.htm [2006 febrero 20].

Bond, T. (2003). Validity and assessment: a Rasch measurement perspective. Metodología de las Ciencias del Comportamiento, 5 (2), 179-194.

Brody, H. (2000). Mind over medicine. Psychology Today. Nueva York, julio/agosto. 
Burga, A. (2001). Construcción, confiabilidad y validez de la Escala de Respuesta al Estrés-MNC. Tesis para optar la licenciatura en Psicología. Universidad de Lima.

Burga, A. (2003). Escala de Respuesta al Estrés-MNC. Lima: Universidad Peruana Cayetano Heredia.

Calvo, M.; Szabo, A. \& Capafons, J. (1996). Anxiety and heart rate under psychological stress: The effects of exercise-training. Anxiety, Stress and Coping: An International Journal, 9(4), 321-337.

Czeisler, C. \& Richardson, G. (1998). Disorders of sleep and circadian rhythms. En: A. Fauci et al. (Eds.). Harrison's principles of internal medicine, $14{ }^{\text {a }}$ edición. Nueva York: McGraw-Hill.

Daroff, R. \& Martin, J. (1998). Faintness, syncope, dizziness and vertigo. En A. Fauci et al. (Eds.). Harrison's principles of internal medicine, 14. ${ }^{\mathrm{a}}$ edición. Nueva York: McGraw-Hill.

Ficek, S. \& Wittrock, D. (1995). Subjective stress and coping in recurrent tension-type headache. Headache, 35(8), 455-460.

Gutiérrez, J. M. (1998). La promoción del estrés. Psicologia.com [Online], 2(1), 38 párrafos.

Huber, G. (1980). Stress y conflictos. Métodos de superación. Madrid: Paraninfo.
Kahn, R. \& Byossiere, P. (1995). The stress in organizatins. En D. Kolb et al. The organizational behavior reader, $6 .^{\text {a }}$ edición. Indianápolis: Prentice Hall.

Keltikangas-Jaervinen, L.; Kettunen, J.; Ravaja, N. \& Naeaetaenen, P. (1999). Inhibited and disinhibited temperament and autonomic stress reactivity. International Journal of Psychophysiology, 33(3), 185-196.

Lazarus, A. (1993). From psychological stress to the emotions: A history of changing outlooks. Annual Review Psychology, 44, 1-21.

Lazarus, A. \& Folkman, S. (1986). Estrés y procesos cognitivos. Barcelona: Martínez Roca.

Lehrer, P. \& Woolfolk, R. (1993). Specific effectos of stress management techniques. En P. Lehrer \& R. Woolfolk (Eds.). Principles and practices of stress management, $2{ }^{\mathrm{a}}$ edición. Nueva York: The Guilford Press.

Linacre, M. (2001). Category, step and threshold: Definitions \& disordering. Rasch measurement transactions, 15 (1), 794. [En línea]. http://rasch.org/rmt/rmt151 g.htm, [2006, febrero 20].

Linacre, M. (2005). A user's guide to winstpes and ministeps. Raschmodel computer programs. Chicago: Winsteps. 
Linacre, M. (2006). Winsteps Rasch measurement. [Programa informático]. Chicago.

Mandler, G. (1993). Thought, memory and learning: Effects of emotional stress. En L. \& S. Golberger, Breznitz. Handbook of stress. Theoretical and clinical aspects, 2. ${ }^{\mathrm{a}}$ edición. Nueva York: The Free Press.

Meerlo, P.; Van Den Hoofdakker, R. H., Koolhaas, J. M. \& Daan, S. (1997). Stress-induced changes in circadian rhythms of body temperature and activity in rats are not caused by pacemaker changes. Journal-ofBiological-Rhythms, 12(1): 80-92.

Mendl, M. (1999). Performing under pressure: Stress and cognitive function. Applied-Animal-BehaviourScience, 65(3): 221-244.

Muñiz, J. (1997). Introducción a la teoría de respuesta a item. Madrid: Pirámide.

Ogden, J. (1998). Health psychology. A textbook. Philadelphia: Open University Press.

Pruessner, J.; Hellhammer, D. \& Kirschbaum, C. (1999). Low selfesteem, induced failure and the adrenocortical stress response. Personality and individual differences, 27(3), 477-489.

Revelle, W. \& Loftus, D. (1992). The Implications of arousal effects for the study of affect and memory. En
S. A. Christianson (Ed.). (1992). Handbook of emotion and memory. Nueva York: Erlebaum.

Sandin, B. (1995). El estrés. En A. Belloch.; B. Sandin \& F. Ramos. Manual de psicopatología, tomo II. Madrid: McGraw-Hill.

Sandin, B.; Chorot, P.; Santed, M. \& Jiménez, P. (1995). Trastornos psicosomáticos. En A. Belloch B. Sandin \& F. Ramos. Manual de psicopatología, tomo II. Madrid: McGraw-Hill.

Selye, H. (1993). History of the stress concept. En L. Golberger y S. Breznitz. Handbook of stress. Theoretical and clinical aspects, 2. edición. Nueva York: The Free Press.

Shaw, F.; Wrigth, B. \& Linacre, M. (1992). Disordered steps?, Rasch. Measurement Transactions, 6 (2), 225. [En línea]. http://www.rasch.org/rmt/rmt62i.htm, [2006, febrero 20].

Skosnik, P.; Chatterton, R.; Swisher, T. \& Park, S. (2000). Modulation of attentional inhibition by norepinephrine and cortisol after psychological stress. International Journal of Psychophysiology, Vol 36(1): 5968.

Smith, J. (1994). Dinámica de la relajación. Gerona: Tikal.

Spielberger, Ch., \& Moscoso, M. (1996). Reacciones emocionales del estrés: 
Ansiedad y cólera. Avances en Psicologia Clinica Latinoamericana, 14, 59-81.

Straus, S. (1998). Chronic fatigue syndrome. En A. Fauci, et al. (Eds.). Harrison's principles of internal medicine, $14{ }^{\mathrm{a}}{ }^{\mathrm{a}}$ edición. Nueva York: McGraw-Hill.

Tuomisto, M. (1997). Intra-arterial blood pressure and heart rate reactivity to behavioral stress in normotensive, borderline and mild hypertensive men. Health Psychology, 16(6), 554-565.
Valdés, M. y De Flores, T. (1990). Psicobiología del estrés. Conceptos $y$ estrategias de investigación. Barcelona: Martínez Roca.

Verlander, L.; Benedict, J. \& Hanson, D. (1999). Stress and sleep patterns of college students. Perceptual and motor skills, 88(3, Pt 1), 893-898.

Wu, M., Adams, R. \& Wilson, M. (1998). ConQuest: Generalized item response modeling software [Programa informático]. Melbourne: ACER. 